\title{
O Discurso Evolucionista e a Prática Involutiva: um Estudo Empírico sobre o Impacto de Mudanças Tecnológicas sobre o Desenho do Trabalho em Call Centers
}

\author{
Marcia Carvalho de Azevedo \\ Miguel Pinto Caldas
}

\begin{abstract}
Resumo
Call centers estão de maneira crescente atraindo a atenção de profissionais e acadêmicos que estudam o desenho e o processo de trabalho. Enquanto muitos dos primeiros tendem a descrever tais centros como organizações orgânicas de alta tecnologia, muitos acadêmicos - sobretudo aqueles da vertente organizacional crítica - revelam uma condição diferente, marcada por desenho mecanicista. Este artigo segue caminho de pesquisa mais crítico, utilizando dados de estudo comparativo de quatro casos, para entender as mudanças do desenho do trabalho ocasionadas pela tecnologia em call centers. Os resultados mostram que o discurso evolucionista é limitado: em geral, a implantação de novas tecnologias em centrais de atendimento tem tornado estas organizações mais mecanicistas. No entanto, em uma das centrais, a nova tecnologia foi utilizada para transformar o trabalho na direção oposta, criando uma estrutura mais orgânica, sugerindo que não apenas o componente tecnológico, mas também a concepção do trabalho por parte da gerência e a forma de implementação do aparato tecnológico, determinam a evolução ou involução do desenho do trabalho em call centers.
\end{abstract}

Palavras-chave: centrais de atendimento; novas tecnologias; estrutura organizacional.

\begin{abstract}
Call centers are increasingly catching the attention of practitioners and academic researchers studying work design and labor processes. While the former tend to depict such centers as hightech, organic organizations, the latter - at least those with a more critical view of organizational reality - seem to be unveiling a different condition, marked by a mechanicist design. This paper follows a more critical stream of research, using data from a four case comparative study to understand work design transformation brought on by technology in call center environments. Results indicated that the evolutionist discourse is limited: in general, technology implementations in call centers investigated led to more machanicist organizations. However, at one center, the new technology was used to transform work in the opposite direction, leading to a more organic structure. Hence, the study reinforces the argument that not only technology, but also the manager conception of work and how such technology is implemented, are important to determine the evolution or involution of work design at call centers.
\end{abstract}

Key words: call centers; new technologies; organizational structure. 


\section{INTRODUÇÃo}

Ao longo das últimas décadas, temos assistido a mudanças profundas no funcionamento das organizações. Podemos identificar a existência de um discurso corrente que apresenta as características necessárias para as empresas vencerem dentro de um novo ambiente competitivo. Este discurso propõe que, em geral, a inovação tecnológica estaria propiciando o nascimento de organizações modernas e que estas novas empresas estariam substituindo organizações ultrapassadas, não adequadas para atuar no novo cenário.

Já no início do século XXI surge novo formato organizacional em uma economia fortemente baseada no setor de serviços (HAMEL; PRAHALAD, 1996; HANDY, 1985; TOFFLER, 1985). Esta argumentação gerencialista caracterizase pela apresentação de formatos organizacionais futuristas como evoluções de modelos anteriores ultrapassados. Este discurso propõe também novo modelo de gestão de pessoas; assim, dentro das organizações flexíveis, encontraríamos novas formas de organização do trabalho.

Esta nova organização flexível demandaria novo tipo de trabalhador com mudanças em termos de requisitos e responsabilidades. $\mathrm{O}$ antigo trabalhador industrial braçal estaria sendo substituído por um trabalhador do conhecimento. Este discurso prega uma transformação da estrutura dos cargos na direção de tarefas mais significativas e interessantes, ou seja, estaríamos assistindo ao fim do trabalho fragmentado e repetitivo (PETERS; WATERMAN, 1982).

Tal modelo substituiria a estrutura industrial tradicional, trazendo organicidade às empresas tayloristas/fordistas, em que as tarefas são fixas e simplificadas, deixando para trás um ambiente com supervisão intensa e sem autonomia. No entanto, ao observamos mais de perto a realidade, vemos um cenário um pouco diferente; podemos constatar, em diversos contextos organizacionais, a permanência e o aprimoramento de modelos tradicionais.

O ambiente de call centers é um segmento que tem sido profundamente afetado por mudanças tecnológicas, e alguns artigos na área administrativa (CALLAGHAN; THOMPSON, 2002; CREAGH, 1998; SILVA et al., 2002) vêm discutindo a forma de estruturação do trabalho nas modernas centrais de atendimento. Dentro dessas centrais, a utilização de avanços tecnológicos tem alterado radicalmente sua forma de funcionamento. Nesses ambientes, o desenho do trabalho tem sido modificado na direção de maior fragmentação e rigidez da tarefa de atendimento. 
O mundo real parece apontar para a limitação da profetizada organização moderna e das novas relações de trabalho (GALEÃO-SILVA; AQUINO, 2002). Até mesmo a mudança de espaço físico dentro das organizações, que muitas vezes é apresentada como elemento de apoio em processos de mudança organizacional que buscam maior flexibilidade, na prática permite controle muito maior dos funcionários (ALCADIPANI; ALMEIDA, 2000). No mundo dos call centers, a utilização de sofisticadas tecnologias parece ter propiciado a permanência de modelos organizacionais, a princípio ultrapassados e fora de moda. O discurso gerencialista tende a dizer exatamente o contrário: a inovação tecnológica seria o combustível necessário para a evolução organizacional. A princípio, pelo menos no que transparece no mundo da praxis, call centers modernos seriam um ambiente propício para os princípios da nova organização emergente, ou seja, as modernas centrais de atendimento teriam um funcionamento mais orgânico do que, por exemplo, as tradicionais organizações fabris ou mesmo as antigas centrais de atendimento. A falta de formalização teórica desse pressuposto, contra o qual este artigo se coloca, será problematizada na seção final do artigo.

A contraposição desses dois tipos ideais de desenho de trabalho, o tipo orgânico e o mecânico sugerem uma pergunta de pesquisa importante: será que a alta tecnologia introduzida nesses tipos 'modernos' de organização, como é o caso dos call centers, provoca mudanças no desenho do trabalho no sentido de fazê-lo mais orgânico, como sugere o discurso gerencialista (e.g., HANDY, 1985; REICH, 1992), ou no sentido de fazê-lo mais taylorizado, como sugere a literatura mais crítica (e.g., FERNIE; METCALF, 1998; FRENKEL et al., 1998; SILVA et al., 2002; TAYLOR; BAIN, 1999)?

Pensando nessa questão, a presente pesquisa teve como objetivo investigar empiricamente qual tem sido o impacto das novas tecnologias na estrutura de trabalho dos call centers. O estudo observou variáveis clássicas e bem aceitas: por um lado, para analisar estrutura organizacional, focamos duas dimensões estruturais, formalização e centralização, usando as medidas sugeridas pelo Grupo de Aston (PUGH; HICKSON, 1976; DONALDSON et al., 1975); por outro lado, para entender o elemento rotina, utilizamos o conceito de tecnologia apresentado por Perrow (1967). A pesquisa coletou dados por meio de estudo comporativo de quatro casos, buscando entender a intensidade e direção da transformação do desenho do trabalho ocasionada pela tecnologia em ambientes de call centers. Dados primários foram coletados, usando uma metodologia mista de pesquisa (CRESWELL, 2003), incluindo questionários, entrevistas, observação direta e análise documental, bem como técnicas projetivas (desenhos). 
O restante do artigo está organizado da seguinte forma: na seção seguinte, revisamos rapidamente a teoria de fundo do trabalho, incluindo a discussão sobre a relação tecnologia $\mathrm{x}$ desenho do trabalho, e a emergência dos modernos call centers. Em seguida, a pesquisa é descrita dos métodos aos resultados. Na seção posterior, faz-se breve discussão dos resultados e, na seção final, o trabalho é concluído pondo em perspectiva os achados e sugerindo caminhos de investigação futura.

\section{TEORIA}

\section{Teorias sobre a Relação entre Tecnologia e Desenho do Trabalho}

A argumentação de que o avanço tecnológico propiciaria a evolução das organizações para modelos de funcionamento mais orgânicos encontra apoio em diversos trabalhos que tratam do tema tecnologia e estrutura organizacional (HAMEL; PRAHALAD, 1996; TOFFLER, 1985).

Estudos acadêmicos clássicos sobre o impacto da mudança tecnológica no funcionamento das organizações (PERROW, 1967; PUGH; HICKSON, 1976; WOODWARD, 1965) identificaram o sistema tecnológico como fator fundamental para a formatação da estrutura organizacional. O trabalho de Joan Woodward (1965) propôs que o avanço da tecnologia faria surgir primeiro uma estrutura mais mecanicista e, em grau mais avançado, uma mais orgânica. Na década de 60, um grupo de pesquisadores, que ficou conhecido como Grupo de Aston (DONALDSON et al., 1975; PUGH; HICKSON, 1976), realizou ampla pesquisa relacionando organizações e meio ambiente, para identificar dimensões da estrutura organizacional. Diversas pesquisas, utilizando a metodologia proposta pelo Grupo de Aston, foram conduzidas. De acordo com Child (1974), tem surgido uma concordância de que as três principais dimensões da estrutura organizacional são: (1) centralização, que é a extensão com que a autoridade de tomar decisões está confinada a níveis superiores da hierarquia, abrangendo os conceitos de autonomia e participação; (2) especialização, que é a extensão em que os deveres oficiais estão divididos entre as áreas funcionais e dentro delas; e (3) formalização, que abrange os conceitos bastante relacionados de Pugh e Hickson (1976) de padronização (a extensão na qual as atividades estão sujeitas a procedimentospadrão) e formalização (a extensão na qual procedimentos, regras e instruções estão escritos). 
Por fim, dos estudos clássicos vale destacar o modelo de Perrow (1967) como referência para a dimensão de rotina. Perrow relacionou rotina do trabalho com tecnologia, propondo um modelo que identificou dois aspectos como diretamente relevantes para a estrutura organizacional. O primeiro é o número de exceções que ocorrem no trabalho; e o segundo diz respeito à natureza do processo de procura que é realizado pelo indivíduo quando as exceções ocorrem. Perrow teorizou acerca de que esses aspectos são dimensões tecnológicas inter-relacionadas e propôs a existência de uma diagonal de rotina/não-rotina. Apesar de a definição de tecnologia proposta no modelo de Perrow (1967) não abarcar todos os elementos do tipo de tecnologia utilizado em call centers, o nível de rotinização é um conceito que se mostra bastante adequado para analisar as atividades realizadas nesses ambientes.

Para o estudo da variável tecnologia este trabalho utilizou como base o Manual de Frascati da OCDE (1993), que define inovação científica e tecnológica como a transformação de uma idéia em: produto novo ou melhorado introduzido no mercado; ou processo de fabricação novo ou melhorado utilizado na indústria ou no comércio; ou em novo enfoque de serviço social. Nesta pesquisa, foi considerado que houve mudança tecnológica, quando ocorreu mudança no processo de trabalho decorrente da alteração de máquinas, equipamentos e programas de computador, que os funcionários utilizam para realizar o seu trabalho.

O trabalho de Zuboff (1988) trouxe elementos importantes para a discussão da relação entre a tecnologia e o desenho do trabalho. A autora, através de amplo e longo estudo em diversos tipos de organizações, identificou que o impacto da implantação de novas tecnologias é mediado em grande proporção pela forma com que esta implementação ocorre.

\section{Call Centers}

Call centers modernos são ambientes cujo funcionamento é baseado em tecnologias das áreas de informação, computação e telecomunicações. O progresso tecnológico levou a um entrelaçamento cada vez maior dessas três áreas e, em particular, propiciou o desenvolvimento e consolidação dos call centers. Devido ao seu ambiente de funcionamento, eles são locais que se caracterizam pela mudança tecnológica constante. Os call centers não são fenômeno novo; as antigas centrais de atendimento foram precursoras dos modernos call centers. Diversos artigos (CALLAGHAN; THOMPSON, 2002; CREAGH, 1998) sobre este tipo de organização descrevem uma mudança tecnológica grande no funcionamento dessas empresas. 


\section{Tecnologia e Desenho do Trabalho em Call Centers}

Ao olharmos mais de perto o funcionamento dos call centers, percebemos que a inserção de mudanças tecnológicas nesses ambientes tem acarretado uma realidade complexa.

\section{Quadro 1: Características da Organização Industrial X Ideal da “Organização Flexível” X Call Center Moderno}

\begin{tabular}{|c|c|c|c|}
\hline & $\begin{array}{c}\text { Organização } \\
\text { Industrial }\end{array}$ & $\begin{array}{c}\text { Idealização da } \\
\text { “Organização Flexível” } \\
\end{array}$ & Modelo do Call center Moderno \\
\hline $\begin{array}{l}\text { Estrutura da } \\
\text { tarefa }\end{array}$ & Pré-determinada & . Em transformação & . Pré-determinada \\
\hline $\begin{array}{l}\text { Desenho dos } \\
\text { processos }\end{array}$ & $\begin{array}{l}\text { Formalizado } \\
\text {. Rígido } \\
\text { Exógeno }\end{array}$ & $\begin{array}{l}\text { Informal } \\
\text { Flexível } \\
\text { Endógeno }\end{array}$ & $\begin{array}{l}\text { Formalizado (pelo sistema) } \\
\text { Rígido (script) } \\
\text { Exógeno (operador não } \\
\text { influencia desenho da tarefa) }\end{array}$ \\
\hline $\begin{array}{l}\text { Desenho do } \\
\text { cargo }\end{array}$ & $\begin{array}{l}\text { Estreito } \\
\text { Trabalho } \\
\text { desqualificado } \\
\text { (baixa exigência } \\
\text { de habilidades) }\end{array}$ & $\begin{array}{l}\text { Amplo } \\
\text { Multifuncionalidade } \\
\text { Exigência de um } \\
\text { "trabalhador do } \\
\text { conhecimento" }\end{array}$ & $\begin{array}{l}\text { Estreito } \\
\text { Trabalho semi-qualificado } \\
\text { (baixa exigência de habilidades } \\
\text { além do domínio do sistema) }\end{array}$ \\
\hline Hierarquia & $\begin{array}{l}\text { Vertical } \\
\text { Formal } \\
\text { Bem definida }\end{array}$ & $\begin{array}{l}\text { Mínima } \\
\text {. Informal } \\
\text {. Base maior em times do } \\
\text { que em estrutura de } \\
\text { autoridade }\end{array}$ & $\begin{array}{l}\text {. Vertical } \\
\text {. Formal } \\
\text {. Bem definida }\end{array}$ \\
\hline $\begin{array}{l}\text { Supervisão } \\
\text { do trabalho }\end{array}$ & $\begin{array}{l}\text { Supervisão estrita } \\
\text { Intenso controle } \\
\text { de produto e } \\
\text { processo }\end{array}$ & $\begin{array}{l}\text { Supervisão branda } \\
\text {. Baseada em resultados } \\
\text { Autocontrole }\end{array}$ & $\begin{array}{l}\text { Supervisão estrita e intensiva } \\
\text { (monitoração de chamadas, } \\
\text { vigilância eletrônica) } \\
\text { Intenso controle de produto e } \\
\text { processo (aderência a script, } \\
\text { conformidade a padrão de } \\
\text { comportamento prescrito) }\end{array}$ \\
\hline $\begin{array}{l}\text { Relação } \\
\text { Chefe - } \\
\text { Subordinado }\end{array}$ & $\begin{array}{l}\text { Baseada em } \\
\text { desconfiança } \\
\text { Separação } \\
\text { concepção x } \\
\text { execução } \\
\text { Decisão no topo } \\
\end{array}$ & $\begin{array}{l}\text { Baseada em confiança } \\
\text { Trabalhador participa } \\
\text { ativamente do } \\
\text { planejamento do } \\
\text { próprio trabalho } \\
\text { Decisão participativa }\end{array}$ & $\begin{array}{l}\text { Baseada em desconfiança } \\
\text { (monitoramento) } \\
\text { Forte separação concepção x } \\
\text { execução } \\
\text { Decisão centralizada em quem } \\
\text { programa o sistema }\end{array}$ \\
\hline
\end{tabular}

Fonte: Azevedo e Caldas, 2002.

Conforme discutido no início deste artigo, o discurso gerencialista aponta o nascimento de organizações flexíveis e orgânicas (HANDY, 1985). Nesses ambientes organizacionais, o trabalho privilegiaria a multifuncionalidade e o conhecimento dos funcionários. O velho modelo industrial, com estruturas rígidas, tarefas fragmentadas e hierarquia rígida estaria sendo substituído pelas novas organizações do século XXI. 
Dentro dos call centers, pesquisas recentes (CALLAGHAN; THOMPSON, 2002; CREAGH, 1998; VASCONCELOS et al., 2001) têm mostrado que o tipo de atividade desenvolvida nessas organizações não exige, em geral, funcionários com experiência prévia. A estrutura das tarefas, normalmente simples e repetitivas, faz com que conhecimentos específicos não sejam um fator importante. É por esse motivo que o trabalho na indústria de call centers tem sido comparado aos tradicionais sweatshops (CALLAGHAN; THOMPSON, 2002; SILVA et al., 2002; TAYLOR; BAIN, 1999;). Kobielus (1994) também vê semelhanças entre os call centers e os sweatshops: o trabalho é incessante, repetitivo e sem fim. De fato, ao focarmos a estrutura do trabalho dos atendentes de call center, percebemos que as mudanças tecnológicas têm permitido uma flexibilização do onde realizar o trabalho, mas não necessariamente do como este trabalho deve ser realizado. Quando analisamos o desenho do trabalho, o que percebemos é que as inovações na área de informática e telecomunicações têm sido aplicadas de forma a aumentar a fragmentação, simplificando a tarefa de atendimento.

O Quadro 1 apresenta, resumidamente, uma comparação das principais características desses diferentes tipos de organização de acordo com alguns artigos (CALLAGHAN; THOMPSON, 2002; FERNIE; METCALF, 1998; SILVA et al., 2002; TAYLOR; BAIN, 1999). O resultado aponta uma semelhança significativa entre os call centers modernos e a organização industrial tradicional. Vasconcelos et al. (2001) identificaram a existência de contradições e paradoxos na utilização de modelos por parte das empresas, para justificar a implementação de novas tecnologias em centrais de atendimentos. Temos, portanto, aparentemente a permanência do velho modelo industrial, agora adaptado para organizações de serviço. Ao compararmos o funcionamento dos novos call centers com as antigas centrais de atendimento, identificamos um perfil de trabalho radicalmente diferente. Os atendentes tinham muito mais autonomia, pois não existiam monitoria nem gravação dos atendimentos. Como os relatórios de desempenho eram simplificados, o controle sobre o trabalho era muito menor. As tarefas não eram formalizadas pela utilização de scripts, nem eram tão fragmentadas pela utilização de tecnologia.

É de certa forma assustador constatar que as antigas centrais de atendimento funcionavam de forma menos mecanicista que os modernos call centers. Neste tipo de organização parece estar ocorrendo exatamente o contrário do preconizado por pesquisas sobre tecnologia e estrutura organizacional (PUGH; HICKSON, 1976; WOODWARD, 1965), com o uso intensivo de tecnologia que favorece um funcionamento mais mecânico, em vez do modelo orgânico idealizado em textos mais recentes (HANDY, 1985; TOFFLER, 1985).

Nosso interesse de pesquisa foi justamente investigar a adequação deste modelo organicista, idealizado pelo discurso gerencial moderno, para a realidade dos call centers. 


\section{Estudo Empírico}

\section{Variáveis e Métodos de Pesquisa}

Como já mencionado, a presente pesquisa objetivou investigar empiricamente o impacto das novas tecnologias na estrutura de trabalho dos call centers: se no sentido do tipo ideal organicista, como sugere o discurso gerencialista hegemônico, ou se no sentido mecanicista, como sugere a literatura mais crítica. A pesquisa compreendeu um estudo comparativo de quatro casos, buscando entender a direção e intensidade da transformação do desenho do trabalho ocasionada pela tecnologia no ambiente dos call centers.

Para a análise dos elementos de estrutura organizacional e desenho do trabalho, utilizamos o direcionamento de Child (1974), e decidimos estudar especificamente duas das três dimensões estruturais clássicas: nível de formalização e nível de centralização. O nível de centralização tem sido investigado a partir de duas dimensões relacionadas: autonomia e participação na tomada de decisões. Entendemos que a dimensão de especialização não seria essencial para este trabalho, já que o nosso foco é primariamente o estudo de apenas uma função nas centrais de atendimento: os atendentes. Para investigação do nível de formalização, foi utilizado o instrumento proposto por Martin e Glisson (1989), cuja escala é uma adaptação da apresentada por Hall (1963) para a mesma variável. Já o nível de centralização foi levantado através do instrumento de Hall (1963), o qual mensura esta variável através de duas escalas: uma de autonomia e outra de participação. Essas escalas abarcam dimensões complementares da variável centralização. Esse instrumento foi identificado como válido e preciso no estudo realizado por Dewar et al. (1980) e utilizado em diversas pesquisas que investigaram a estrutura organizacional (AIKEN; HAGE, 1968; GLISSON; MARTIN, 1980). Para a observação dos elementos ligados à rotina, foi utilizada a escala desenvolvida por Hall (1963), que na literatura funcionalista clássica é aceita como medida da dimensão de exceções do modelo proposto por Perrow. Esse instrumento foi utilizado em diversos estudos (AIKEN; HAGE, 1968; GLISSON; MARTIN, 1980), e validado posteriormente por Dewar et al. (1980).

\section{Seleção da Amostra e Coleta de Dados}

O critério para que uma empresa fizesse parte deste estudo foi que ela tivesse uma central de atendimento aos consumidores, própria ou terceirizada. Esta pesquisa estudou ambientes de call center que passaram por processos de 
introdução de novas tecnologias, através da mudança de máquinas, equipamentos ou programas de computador, que alteraram, de forma substancial, o desenho da tarefa dos atendentes. Esse processo deveria ter ocorrido no máximo nos últimos cinco anos, e funcionários que vivenciaram o processo teriam que estar disponíveis para serem entrevistados. A pesquisa foi realizada em quatro empresas que atendiam a esse seletivo protocolo.

Decidimos estudar a estrutura organizacional e o desenho do trabalho a partir da perspectiva do grupo de atendentes, pesquisando a percepção dos funcionários a respeito de mudanças no seu trabalho após a introdução de inovações. Todas as pessoas que participaram do estudo deveriam ter trabalhado no atendimento antes e depois da introdução das novas tecnologias.

Uma parte substancial da coleta de dados foi realizada a partir de entrevistas individuais com os atendentes e supervisores. Focamos aspectos objetivos e subjetivos envolvidos nos processos de mudança tecnológica. Quanto aos instrumentos, fizemos uso dos dois tipos de metodologias: quantitativas e qualitativas. Os resultados qualitativos foram enriquecidos com a utilização de resultados quantitativos provenientes de um questionário aplicado durante a entrevista semi-estruturada. Classificamos, portanto, o procedimento de coleta de dados como o de métodos mistos, com uma estratégia de triangulação concorrente (CRESWELL, 2003). Nesse tipo de pesquisa, utilizam-se dois métodos diferentes de coleta de dados, na tentativa de confirmar, validar de forma cruzada e corroborar resultados de um estudo. A coleta de dados quantitativos e qualitativos ocorre de forma simultânea, em apenas uma fase de pesquisa. Os resultados dos dois métodos são integrados durante a fase de interpretação.

O levantamento dos dados com os funcionários foi feito em duas etapas principais: entrevistas individuais e observação. Inicialmente foi realizada uma entrevista com pessoas de hierarquia superior que ocupavam posições de gerência. Durante essa entrevista foram levantados dados gerais sobre a empresa. A entrevista foi semi-estruturada, sendo que o roteiro utilizado foi uma adaptação do instrumento de Pugh e Hickson (1976). A coleta dos dados objetivos junto aos atendentes foi feita através de questionários, que focaram os elementos de tecnologia e estrutura organizacional, usando as variáveis clássicas acima mencionadas. Esse levantamento investigou dois períodos distintos: o antes e o depois da implantação de mudanças tecnológicas, sempre usando escalas do tipo Likert. O formato utilizado foi uma escala bipolar com um extremo representando uma resposta negativa e o extremo oposto representando uma resposta positiva. Os valores atribuídos aos diferente itens variavam de $-2 \mathrm{a}+2$.

A coleta de dados subjetivos foi feita durante as entrevistas individuais e durante as observações do ambiente de trabalho. Ao longo das respostas às escalas, 
investigou-se o porquê das opções escolhidas pelos entrevistados. Depois do término do questionário, foi dada uma folha em branco ao entrevistado e pedido a ele que fizesse dois desenhos de como ele se sentia no seu ambiente de trabalho, um representando o ambiente antes, e um o depois da adoção de novas tecnologias. Os comentários dos entrevistados sobre o significado dos desenhos foram anotados nos questionários.

O presente estudo também fez uso da observação simples. Essa observação teve como objetivo a complementação dos dados coletados nas entrevistas, sendo o seu foco o desenho da tarefa dos atendentes e a interação social dentro do ambiente de trabalho.

\section{Amostra}

A pesquisa foi realizada em quatro empresas distintas, em duas cidades. As empresas participantes foram as seguintes: uma montadora de automóveis, uma seguradora, uma distribuidora de derivados de petróleo e uma concessionária de serviços públicos. Classificamos as centrais em dois grandes grupos: receptivas, nas quais os atendentes apenas recebem ligações; e ativas, nas quais os atendentes fazem e recebem ligações. As ligações podem envolver apenas a prestação de serviços, ou agregar também funções de venda. As centrais da montadora e da concessionária se caracterizavam como receptivas de serviços. Já a seguradora realizava também atividades ativas de serviços e a distribuidora, atividades receptivas de vendas. Foram realizadas ao todo 69 entrevistas semi-estruturadas, com atendentes, supervisores e pessoal de apoio. Os sistemas tecnológicos dos call centers foram estudados através dos relatos de atendentes, ex-atendentes, supervisores e funções de apoio. A seguir apresentamos um resumo das características dos call centers pesquisados.

\section{Quadro 2: Resumo das Características dos Call Centers}

\begin{tabular}{|l|c|c|c|c|c|c|c|}
\hline Empresa & $\begin{array}{c}\text { Trab./ } \\
\text { PA's }\end{array}$ & $\begin{array}{c}\text { Ligações } \\
\text { mês }\end{array}$ & $\begin{array}{c}\text { Ritmo de } \\
\text { trabalho }\end{array}$ & Mudança & $\begin{array}{c}\text { Demis- } \\
\text { sões }\end{array}$ & Situação & $\begin{array}{c}\text { Papel no } \\
\text { faturamento }\end{array}$ \\
\hline \hline Seguradora & $60 / 30$ & 45.000 & Intenso & Grande & Poucas & $3^{\circ}$ & Apoio à venda \\
\hline Concessionária & $270 / 156$ & 465.000 & Intenso & Grande & Sim & Próprio & Centro de custo \\
\hline Montadora & $19 / 10$ & 14.500 & Não-int. & Pequena & Não & $3^{\circ}$ & Centro de custo \\
\hline Distribuidora & $47 / 47$ & 50.000 & Não-int. & Grande & Poucas & Próprio & Centro de receita \\
\hline
\end{tabular}

Podemos dividir as empresas em dois grupos distintos quanto ao ritmo de trabalho: montadora e distribuidora - ritmo não-intenso; seguradora e concessionária - ritmo intenso. Apenas a seguradora tem suas atividades de atendimento terceirizadas e operadas fora da sede da empresa. Deve-se ressaltar que as empresas que passaram por mudanças tecnológicas mais abrangentes foram aquelas que centralizaram suas atividades. A ocorrência de demissões foi 
mais intensa em apenas um dos call centers estudados; entretanto, apesar de terem ocorrido demissões nessa empresa, houve simultaneamente um número grande de contratações, decorrente do aumento do quadro de atendentes. No que se refere ao papel no faturamento da empresa, de um lado temos centrais que têm um papel de prestação de serviços e funcionam como centros de custo. De outro lado, temos centrais que têm também um papel no faturamento da empresa: uma delas presta apoio à venda, e a outra funciona como canal de vendas propriamente dito.

Inicialmente, decidimos classificar os sistemas tecnológicos das diferentes centrais de atendimento. Devido à inexistência de uma classificação tecnológica padrão para centrais de atendimento, fizemos um levantamento de dimensões dos componentes tecnológicos das centrais que afetam a estrutura do trabalho. Foram identificadas quatro dimensões: rotina, formalização, autonomia e nível de controle. Após a análise dos dados levantados, identificamos dentro de cada dimensão diversos parâmetros importantes para uma classificação da tecnologia utilizada. O Quadro 3 apresenta os resultados desta análise.

\section{Quadro 3: Caracterização dos Componentes Tecnológicos de Call Centers}

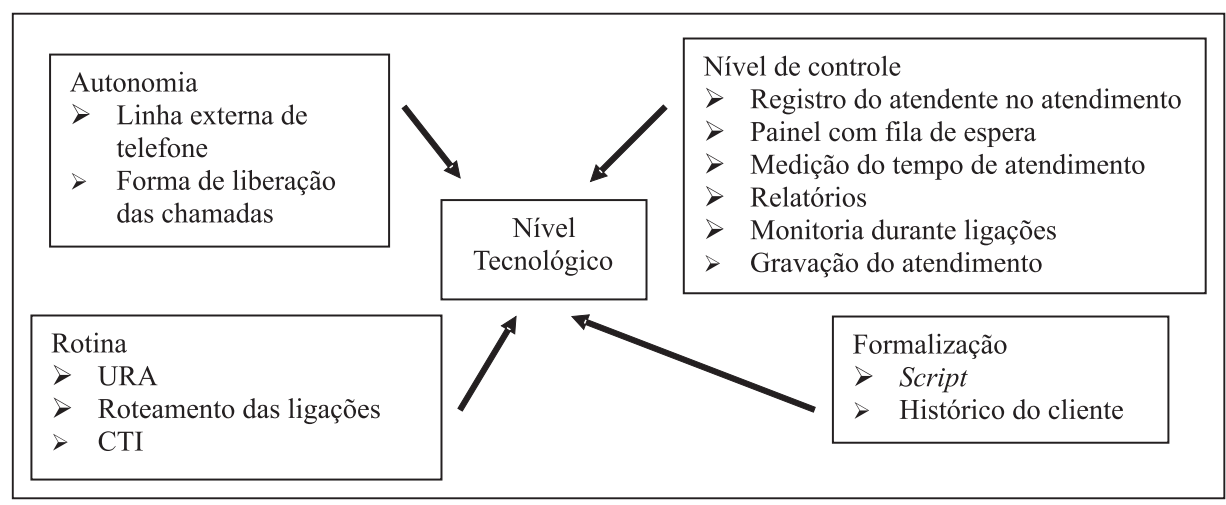

Através dos parâmetros apresentados no quadro anterior, foi feita uma classificação das dimensões em três níveis diferentes. O nível 1 tendo uma menor intensidade de utilização de recursos tecnológicos, e o nível 3 sendo aquele com maior sofisticação tecnológica. Para identificar o nível de cada uma das dimensões nas diferentes centrais de atendimento, foi feita uma classificação final de cada empresa, antes e depois do processo de mudança estudado.

A classificação foi feita a partir dos dados quantitativos e qualitativos obtidos durante a pesquisa. Identificamos também (ver Quadro 4) a direção e a intensidade da mudança. Classificamos o uso de máquinas, equipamentos e programas 
computacionais mais avançados tecnologicamente, como uma mudança na direção de evolução, e o uso de recursos mais simples como uma involução. A intensidade da mudança foi atribuída a partir da mudança de nível das dimensões tecnológicas (rotina, formalização, autonomia e nível de controle), decorrente de mudanças nos parâmentros associados a cada dimensão.

\section{Quadro 4: Caracterização das Mudanças Tecnológicas Ocorridas nos Call Centers Estudados}

\begin{tabular}{|l|c|c|c|c|}
\hline Empresa & Antes & Depois & Direção da mudança & Intensidade \\
\hline \hline Seguradora & Nível 2 & Nível 3 & Evolução & Alta \\
\hline Concessionária & Nível 2 & Nível 3 & Evolução & Alta \\
\hline Montadora & Nível 2 & Nível 2 & Involução & Baixa \\
\hline Distribuidora & Nível 1/Nível 2 & Nível 2 & Evolução & Média \\
\hline
\end{tabular}

Podemos observar que houve mudanças nos níveis de configuração dos sistemas tecnológicos em três das quatro centrais estudadas. A montadora passou por um processo de reformas e com isso alguns recursos tecnológicos foram desativados; classificamos portanto a mudança como involução. No entanto a modificação foi pequena, o que fez com que esta empresa mantivesse o mesmo sistema tecnológico, permanecendo no nível 2. A seguradora e a concessionária tiveram mudança do nível 2 para o nível 3; classificamos as mudanças como evolução de intensidade alta. Já a distribuidora sofreu mudança de um nível classificado como 1.5 para o nível 2. Neste caso entendemos que houve também uma evolução do sistema tecnológico, no entanto a intensidade foi média.

\section{Resultados}

O questionário mediu três variáveis relacionadas com a estrutura da organização: rotina, formalização e centralização. Essas variáveis foram mensuradas através de 21 itens do tipo Likert. A variável de rotina foi investigada em 5 respostas; a de formalização em 7 e a de centralização em 9. No presente estudo, a hipótese inicial era de que a introdução de novas tecnologias no ambiente dos call centers tem levado as organizações na direção de modelos mais mecanicistas, com aumento da formalização, centralização e rotina das atividades.

A variável de centralização foi investigada através de perguntas agrupadas em duas dimensões. Denominamos uma dimensão de autonomia e a outra de participação na tomada de decisões. A Tabela 1 apresenta os resultados numéricos gerais divididos por empresa. 


\section{Tabela 1: Resultados das Variáveis de Rotina, Formalização e Centralização dos Call Centers}

\begin{tabular}{|l|c|c|c|c|c|c|c|c|}
\hline & \multicolumn{2}{c}{$\begin{array}{c}\text { Rotina } \\
\text { Quanto }> \\
\text { +rotina }\end{array}$} & $\begin{array}{c}\text { Formalização } \\
\text { Quanto }>\end{array}$ & \multicolumn{2}{c|c}{$\begin{array}{c}\text { Centralização } \\
\text { +formalização }\end{array}$} & $\begin{array}{c}\text { Quanto }> \\
\text { - autonomia }\end{array}$ & $\begin{array}{c}\text { Quanto }> \\
\text { - participação }\end{array}$ \\
\hline & Antes & Depois & Antes & Depois & Antes & Depois & Antes & Depois \\
\hline Seguradora & 0.3 & 3.2 & 2.8 & 8.2 & 2.8 & 8.0 & 6.0 & 7.0 \\
\hline Concessionária & 2.2 & 3.9 & 4.7 & 8.1 & -0.4 & 0.6 & 6.2 & 4.8 \\
\hline Montadora & 1.3 & 1.8 & 3.3 & 3.3 & -1.8 & -1.8 & 7.3 & 7.0 \\
\hline Distribuidora & 2.3 & -1.0 & 2.8 & 0.9 & -4.0 & -6.0 & 7.4 & 3.1 \\
\hline
\end{tabular}

Os resultados foram totalizados separadamente por empresas e por variáveis. Para cada variável as respostas dos entrevistados para todos os itens foram somadas, chegando-se assim a um número separado por empresa. Os dados numéricos servem de subsídio para uma análise do impacto da mudança de tecnologia na estrutura de trabalho, permitindo uma classificação das mudanças no que se refere a sua intensidade e a sua direção.

A tabela a seguir resume os dados obtidos. Os números apresentados referemse à diferença dos valores, antes e depois da mudança tecnológica, das variáveis de estrutura organizacional. Em três das empresas pesquisadas as mudanças na variável de participação ocorreram na direção oposta da esperada. Utilizamos como critério para considerar que houve mudanças entre as situações antes e depois uma diferença maior que 1.0 entre as medidas das variáveis nos dois momentos. Com o objetivo de obter um valor que resumisse a intensidade e a direção das mudanças, resolvemos somar os valores das variáveis de rotina, formalização e autonomia. Criamos assim um número resumo e apresentamos este resultado em uma coluna. Este número funciona como indicador de intensidade e direção da mudança. Devido ao seu comportamento diferenciado, os resultados da variável participação não foram computados.

\section{Tabela 2: Resumo das Variáveis de Rotina, Formalização e Centralização dos Call Centers}

\begin{tabular}{|c|c|c|c|c|c|c|c|}
\hline & \multicolumn{4}{|c|}{ Variáveis Estruturais } & & & \\
\hline & \multicolumn{4}{|c|}{ Quanto $>+$ mecanicista } & & & \\
\hline & Rotina & Formaliz. & Autonom. & Particip. & $\underset{\mathbf{R}+\mathbf{F}+\mathbf{A}}{\sum \text { de }}$ & $\begin{array}{l}\text { Intensidade } \\
\text { da mudança }\end{array}$ & $\begin{array}{c}\text { Direção da } \\
\text { mudança }\end{array}$ \\
\hline Seguradora & 2.9 & 5.4 & 5.2 & 1.0 & 13.5 & Alta & Mecanicista \\
\hline Concessionária & 1.7 & 3.4 & 1.0 & $-1.4^{*}$ & 6.1 & Média & Mecanicista \\
\hline Montadora & 0.5 & 0.0 & 0.0 & $-0.3 *$ & 0.5 & Ficou igual & Ficou igual \\
\hline Distribuidora & $-3.3^{*}$ & $-1.9 *$ & $-2.0 *$ & $-4.3 *$ & $-7.2 *$ & Média & Orgânica \\
\hline
\end{tabular}


Os resultados das escalas de rotina, formalização, autonomia e participação na tomada de decisões indicam situações bastante diferenciadas nas organizações estudadas. Podemos dividir as empresas em três tipos, no que se refere à intensidade das mudanças. A seguradora passou por mudança muito forte, enquanto na concessionária e na distribuidora a mudança se caracteriza como tendo intensidade média. Entendemos que na montadora não houve diferenças significativas nos valores das variáveis.

Quanto à direção, podemos dividir as empresas em três grupos distintos. Na seguradora e na concessionária ocorreram mudanças na direção prevista por trabalhos como Taylor e Bain (1999), Fernie e Metcalf (1998) e Callaghan e Thompson (2002). A estrutura organizacional mudou, tornando-se mais mecanicista. Na montadora não houve mudança significativa. Já na distribuidora, os resultados foram exatamente opostos ao previsto: aconteceu uma diminuição no valor de todas as variáveis, indicando mudança para uma estrutura mais orgânica.

Deve-se ressaltar no entanto que, com relação à variável de centralização, os resultados apresentaram outro comportamento. Esta variável foi medida através das dimensões de autonomia e participação. A seguradora foi a empresa com menor autonomia; a concessionária e a montadora tiveram resultados parecidos com uma maior autonomia dos funcionários. Em relação à participação, a seguradora e a montadora tiveram menor participação; já a concessionária apresentou um índice maior. A distribuidora manteve os menores valores do grupo para as duas dimensões, indicando baixa centralização.

Parte dos dados qualitativos coletados foram desenhos feitos durante as entrevistas. Este material caracterizou claramente as diferenças existentes nos dois momentos que foram foco do presente estudo. Na seguradora, as figuras escolhidas para simbolizar o antes trazem elementos afetivos e positivos. Já as imagens que representam o momento posterior estão na sua maioria associadas a aspectos negativos.

Na concessionária muitos entrevistados relataram uma percepção de aumento da rotina e da formalização e diminuição na autonomia. Os desenhos desses funcionários transmitem os sentimentos associados a essa mudança. Figuras com grades são recorrentes, e quase todas as vezes que são retratadas pessoas na situação depois, elas são colocadas em situações de sofrimento (prisão) com rosto triste.

Na montadora todos os entrevistados fizeram apenas um desenho, pois achavam que não houve mudanças na forma como eles se sentiam no trabalho. Todas as imagens passam sentimentos positivos e a falta de percepção de mudanças significativas. 
Na distribuidora os desenhos retratam uso de imagens muito diferentes entre si; no entanto podemos identificar alguns temas comuns. De forma geral, o discurso dos entrevistados foi bastante racional, com poucas manifestações de aspectos afetivos. Já o novo sistema foi representado de forma positiva, e o Sol foi tema de dois desenhos. É interessante notar que essa foi a única empresa em que uma pessoa representou um atendente com um sorriso no rosto para a situação após a mudança de sistemas.

Um resumo dos principais resultados pode ser visto no Quadro 5: as mudanças dos componentes dos sistemas tecnológicos e as mudanças na estrutura organizacional. As primeiras colunas apresentam a classificação dos componentes dos sistemas tecnológicos dos diferentes call centers. Quando a diferença dos valores do antes e do depois das variáveis de estrutura é inferior a 1, consideramos que as duas situações são iguais e atribuíamos o sinal =. Quando a diferença é igual ou superior a 1, consideramos que houve mudança; quando o nível posterior é superior ao anterior, atribuíamos à mudança o sinal >; e quando fosse o contrário, atribuímos à mudança o sinal < . Nas duas últimas colunas salienta-se a mudança que era esperada na estrutura e o que aconteceu de fato. Discutiremos os resultados expressos nesse quadro na próxima seção.

\section{Quadro 5: Resumo das Mudanças nos Call Centers}

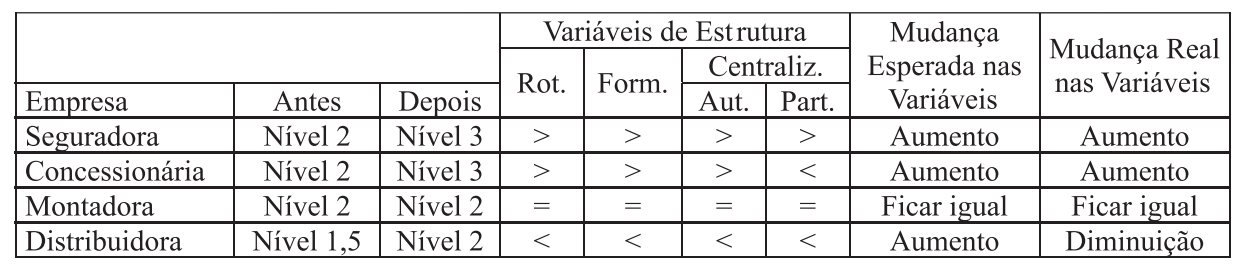

\section{Dıscussão}

De maneira geral, como esperávamos, os elementos tecnológicos estudados tiveram impactos na direção de maior mecanicismo da organização do trabalho, com aumento da rotina, da formalização e da centralização. Porém esses mesmos elementos tecnológicos tiveram sua implantação mediada por diferentes formas de utilização de tecnologia, em cada um dos call centers pesquisados. Isso fez com que a implantação dos mesmos recursos tecnológicos avançados (ex: roteamento das ligações, script, liberação de chamadas) tivesse impacto diferente em um dos call centers analisados, favorecendo um funcionamento mais orgânico. 
Claramente, esperávamos dois tipos de resultados. Em três dos call centers estudados (seguradora, concessionária e distribuidora), em função de ter ocorrido mudança de tecnologia com a utilização de recursos mais avançados tecnologicamente, a expectativa era de que o impacto fosse de involução, gerando maior mecanização da estrutura e da organização do trabalho: ou seja, esperavase aumento no valor das variáveis de estrutura. Já no quarto call center analisado, o da montadora, em função da estabilidade da variável tecnológica, esperávamos que não houvesse impacto nem involutivo nem evolutivo. Pode-se verificar (ver Quadro 5) que, em três dos quatro casos, o que era esperado foi o que ocorreu.

No caso da seguradora, os resultados das entrevistas estão de acordo com o tipo de mudança esperada na estrutura do trabalho. Em razão do aumento de nível do sistema tecnológico do nível 2 para o nível 3, esperávamos que houvesse aumento nas variáveis de rotina, formalização e centralização. Esse foi exatamente o resultado obtido na seguradora, tanto nos resultados numéricos quanto no material dos desenhos.

Os resultados da concessionária foram bastante similares. O sistema tecnológico utilizado pela empresa tornou-se mais avançado e esperávamos que a mudança de tecnologia do nível 2 para o nível 3 tornasse a estrutura mais mecanicista. Esse foi o resultado encontrado para as variáveis de rotina, formalização e autonomia. Já a variável de participação na tomada de decisões mudou na direção oposta da prevista inicialmente. Esse dado pode ser entendido ao levarmos em conta que esta empresa passou por um processo de privatização e com isso houve mudança no seu estilo de gestão na direção de um funcionamento mais democrático.

Os resultados quantitativos e qualitativos das entrevistas da montadora indicam que não houve transformação na estrutura da tarefa; esse era o resultado esperado, já que não houve mudanças no nível da tecnologia utilizada.

No entanto, na distribuidora, aconteceu exatamente o contrário do esperado: prevíamos que a partir da mudança tecnológica que aconteceu (nível 1.5 para nível 2) haveria uma involução (aumento de rotina, de formalização e de centralização), gerando mais mecanização da estrutura e da organização do trabalho. Aconteceu exatamente o contrário: uma redução em todas as variáveis. A análise dos dados qualitativos e numéricos dos questionários apontam para uma percepção da estrutura do trabalho como mais flexível e variada.

O processo de mudança de tecnologia na distribuidora foi explicado detalhadamente durante a coleta de dados junto à gerência. Durante essas entrevistas ficou claro que a proposta de funcionamento do call center da 
distribuidora era radicalmente diferente daquela das outras centrais. Como nas outras empresas, a redução de custos da estrutura foi um dos objetivos da distribuidora, ao centralizar as atividades. A distribuidora, no entanto, teve algumas diferenças marcantes com relação às outras empresas estudadas. Desde o início, a central de atendimento foi estruturada para reposicionar o setor de atendimento no interior da empresa, dentro de novo conceito de atendimento. Houve mudança na concepção do trabalho do atendente que levou a uma redefinição do desenho do trabalho dos funcionários. A tarefa de atendimento foi limitada a algumas horas do trabalho diário. O restante do tempo do atendente foi direcionado para outras atividades. Assim houve diversificação das atividades dos funcionários e ocorreu enriquecimento do trabalho, o que demandou atendentes mais qualificados.

Em todas as organizações pesquisadas, verificamos que a inserção de tecnologia no call center trouxe mudanças nos componentes e no nível de controle sobre o trabalho dos atendentes. Em especial, ocorreu aumento do nível de controle através de maior utilização dos parâmetros dos sistemas tecnológicos: gravação, relatórios, tempo médio de atendimento, nota de atendimento, monitoramento aleatório. No entanto verificamos que a intensidade do controle real está mais ligada ao uso que a empresa faz dos componentes tecnológicos do que à configuração da tecnologia propriamente dita.

Os resultados reforçam a tese de que a intensidade de utilização dos recursos tecnológicos está diretamente ligada aos pressupostos dos atores envolvidos no desenho do trabalho de atendimento. O fato de a distribuidora utilizar controles mais simplificados não é devido a alguma impossibilidade de adquiri-los (por exemplo, questões de ordem financeira ou estrutural) e sim a uma decisão da inadequação de sua utilização.

Com relação à dimensão de autonomia, que é o controle que o atendente tem do ritmo de trabalho, a forma como a tecnologia é utilizada é mais importante do que o recurso tecnológico propriamente dito. Portanto, apesar de o sistema tecnológico ser o mesmo, de acordo com a configuração do equipamento, em algumas centrais, o atendimento é realizado através da liberação automática de chamadas (seguradora e concessionária), favorecendo o mecanicismo, enquanto em outras (montadora e distribuidora) ocorre o oposto, apesar de a possibilidade de atendimento automático também existir.

Ao analisarmos o conhecimento necessário ao atendimento, verificamos que em todas as empresas pesquisadas há tendência de os programas computacionais utilizados no atendimento "absorverem” conhecimento. Entretanto, a quantidade de conhecimento “absorvida” pelos programas variou bastante. Em algumas empresas que optaram por trabalhar com uma mão-de-obra pouco especializada, identificamos aumento na formalização das tarefas dos atendentes, propiciada 
pela absorção de conhecimento pelo programa de atendimento. Em contrapartida, quando o tipo de mão-de-obra escolhido entre as organizações pesquisadas era mais qualificado (distribuidora), a quantidade de informações internalizada nos programas tendia a ser reduzida, levando a uma menor formalização das atividades.

Com relação à rotinização, o cenário é bastante semelhante. Os recursos tecnológicos para a implantação de VRUs, roteamento de chamadas e integração entre computadores e telefonia (CTI) estão presentes na maioria das centrais. Quando a principal preocupação da central era a produtividade, existia a tendência de utilização da VRU e do roteamento para maior fragmentação da tarefa de atendimento; isso foi o que aconteceu na concessionária.

\section{Conclusão}

O estudo sugere que a modernização de call centers de fato provoca profundas mudanças em termos do desenho organizacional e do trabalho; mas tais transformações não condizem com a retórica triunfalista da organização flexível. Os casos investigados mostram que as inovações geralmente acarretam mudanças profundas na divisão do trabalho desses ambientes: as tarefas tendem a tornar-se mais formalizadas, os processos mais padronizados e repetitivos e a supervisão mais intensa. Isso nos faz questionar até que ponto caminhamos, de fato, para uma "utopia flexível”.

No entanto, de acordo com esta pesquisa, apesar de predominante, essa não é a única resultante possível nesse tipo de organização. A pesquisa mostrou que a realidade dos call centers é complexa, com elementos importantes mediando os processos de mudança tecnológica. Apesar de o cenário descrito ser até certo ponto sombrio, os resultados também apontaram para a existência de caminhos alternativos. Em uma das organizações pesquisadas as mudanças ocorreram de forma oposta ao esperado: na distribuidora, apesar de as dimensões do sistema tecnológico terem sido modificadas para favorecer um funcionamento mais mecanicista, o resultado foi inesperado: a estrutura do trabalho dos atendentes tornou-se mais orgânica. Nesse call center, a gerência tinha uma concepção diferenciada de como deveria ser o trabalho dos atendentes; a intenção era favorecer uma estruturação do trabalho a priori mais orgânica. Esse resultado aponta para a necessidade da inclusão da concepção da gerência, em relação à estrutura do trabalho de atendimento, na análise do efeito de tecnologia na organização do trabalho em call centers. Em outras palavras, a concepção ex 
ante do desenho ideal do trabalho da central de atendimento é fator essencial que determina a forma como os sistemas tecnológicos são implementados. Devemos notar, no entanto, que mesmo nesse caso houve a introdução de novos componentes tecnológicos de controle, com aumento da intensidade do controle do trabalho do atendente pela organização, reforçando as teses críticas revistas (e.g., FERNIE; METCALF, 1998; FRENKEL et al., 1998; TAYLOR; BAIN, 1999).

Zuboff (1988) chegou a resultados semelhantes, quando identificou que novas tecnologias podem ser utilizadas de duas formas opostas, para qualificar o trabalhador ou para fazer exatamente o inverso, atuando como instrumento que propicia a desqualificação do trabalho. Foi precisamente isso que parece ter ocorrido nas centrais de atendimento aqui estudadas. Em três delas, as mudanças tecnológicas foram utilizadas na configuração de um trabalho mais mecanizado, rotineiro e formalizado. No entanto, em uma das centrais, o aconteceu exatamente o oposto. A mudança tecnológica apoiou maior qualificação dos funcionários e maior organicidade do desenho da organização e do trabalho. O que podemos reforçar nesta pesquisa, portanto, é a antiga tese (sujeita ao conhecido e disputado terreno da organização do trabalho) de que a forma de utilização de componentes tecnológicos está diretamente ligada à concepção de organização pré-existente. A tecnologia nesse cenário se constitui em condição necessária, mas não suficiente, para a transformação das modernas centrais de atendimento em organizações mais mecanicistas: não é apenas a tecnologia que leva a uma alienação e a uma mecanização no trabalho, mas também a forma como ela é implementada pelas empresas.

Por conseguinte, a julgar pela realidade aqui pesquisada, seria inadequado dizer que, no que se refere a call centers, a organização flexível e orgânica é o único modelo para o qual as organizações estariam evoluindo. Porém, de forma análoga, seria incorreto dizer que essas organizações estão necessariamente involuindo em função da inserção de tecnologia.

Apesar de contributivo à discussão sobre a relação entre implementação de tecnologia e o desenho do trabalho e da estrutura organizacional, este trabalho e suas conclusões têm diversas limitações que precisam ser discutidas e, em futuras pesquisas, superadas. Por exemplo, devido ao tamanho da amostra, os resultados obtidos ainda não são facilmente generalizáveis para outros call centers ou organizações. Há uma série de outras limitações relacionadas ao delineamento da pesquisa, como a utilização de variáveis e métodos funcionalistas e o método transversal de coleta de dados. Outros pontos limitantes foram a coleta de dados em centrais primariamente receptivas e a utilização da percepção das pessoas e não de medidas organizacionais reais (unobstrusive data). A coleta de dados em apenas um momento (da situação antes da mudança e depois da mudança) 
também pode ter gerado distorções nos resultados. Sugerimos que outras pesquisas sejam conduzidas com revisão desses elementos, a fim de superar as limitações identificadas neste estudo.

A despeito dessas limitações, é possível dizer: a presente pesquisa mostrou que o discurso triunfalista hegemônico, que parece via de regra defender que inovações tecnológicas tem propiciado uma evolução das organizações na direção de funcionamento mais orgânico e flexível, é na realidade utópico no que se refere ao contexto de centrais de atendimento. Dentro do universo pesquisado, foi verificado que os call centers de fato têm modificado suas estruturas a partir da introdução de novas tecnologias. Porém, em geral tais transformações têm ocorrido no sentido de maior mecanicismo, exatamente o contrário do pregado pelo discurso gerencialista. Por outro lado, apesar de esse direcionamento genérico tendente à involução do modelo organizacional ter sido consistente em três dos casos analisados, nossa pesquisa também revelou que a involução no sentido mecânico não é imperativa. Verificamos que a utilização de novas tecnologias em call centers pode promover maior organicidade, dependendo fundamentalmente de como essa tecnologia é implementada e utilizada. Nesse sentido, a análise dos resultados obtidos reforçou um argumento já proposto por Zuboff (1988), segundo o qual uma peça fundamental na determinação do impacto da tecnologia na estrutura organizacional é a sua concepção ou sua forma de implementação.

Artigo recebido em 16.10.2003. Aprovado em 14.12.2004.

\section{Bibliografia}

AIKEN, M.;

HAGE, J.

Organizational interdependence and intra-organizational structure. American Sociological Review, Albany, v. 33, n. 6, p. 912-930, dec. 1968.
ALCADIPANI, R.;

ALMEIDA, A. O.A.

O feitiço incluiu o feiticeiro: uma análise sobre a implementação de um escritório aberto. ENCONTRO NACIONAL DA ANPAD, 24., 2000, Florianópolis. Anais... Florianópolis: ANPAD, 2000. 1 CD-ROM. 
AZEVEDO, M.C;

CALDAS, M. P.

Seriam os call centers os sweatshops do século XXI? ENCONTRO DE ESTUDOS ORGANIZACIONAIS, 2., 2002, Recife. Anais... Recife: ANPAD. $1 \mathrm{CD}-\mathrm{ROM}$.

CALLAGHAN, G;

THOMPSON, P.

'We recruit attitude': the selection and shaping of routine call centre labour. Journal of Management Studies, Oxford, v. 39, n. 2, p. 233-254, mar. 2002.

\section{CHILD, J.}

Comments on Reimann and Mansfield's bureaucracy.

Administrative Science Quarterly, Ithaca, v. 19, n. 2, p. 247-250, july 1974.

\section{CREAGH, $M$.}

Call centres - The new assembly lines? Credit Control, Hutton, v. 19, n. 10, p. 17-21, oct. 1998.

\section{CRESWELL, J.W.}

Research design: qualitative, quantitative and mixed methods approaches. 2 th ed. California: Sage Publications, 2003. p. 246.

DEWAR, R. B.;

WHETTEN, D. A.;

BOJE, D.

An examination of the reliability and validity of the Aiken and Hage scales of centralization, formalization, and task routineness. Administrative Science Quarterly, Ithaca, v. 25, n. 1, p. 120128, mar. 1980.
DONALDSON, L.;

CHILD, J.;

ALDRICH, $\mathrm{H}$.

The aston findings on centralization: futher discussion. Administrative Science Quarterly, Ithaca, v. 20, n. 3, p. 453-460, sept. 1975.

FERNIE, S.;

METCALF, D.

(Not) Hanging on the telephone: payment systems in the new sweatshops. London School of Economics and Political Science, Discussion Paper \# 390. 1998.

FRENKEL, S. J. et al.

Beyond bureaucracy? Work organization in call centres. International Journal of Human Resource Management, Cardiff, v. 9, n. 6, p. 957-979, dec. 1998.

GALEÃO-SILVA, L. G.;

ALVES, M.A.

A crítica do conceito de diversidade nas organizações. ENCONTRO DE ESTUDOS ORGANIZACIONAIS, 2., 2002, Recife. Anais... Recife: ANPAD, 2002. 1 CD-ROM.

GLISSON, C., MARTIN, P.

Productivity and efficiency in human service organizations as related to structure, size and age. Academy of Management Journal, Briarcliff Manor, v. 23, n. 1, p. 21-37, mar. 1980. 
HALL, R. H.

The concept of bureaucracy: an empirical reassessment. American Journal of Sociology, Chicago, v. 69, n. 1, p.32-40, july 1963.

HAMEL, G;

PRAHALAD, C. K.

Competing in the new economy: Managing out of bounds. Strategic Management Journal, Chichester, v. 17, n. 3, p. 237-242, mar. 1996.

\section{HANDY, C.}

The future of work: a guide to a changin society. London: Blackwell Publishers: 1985. p. 216.

\section{KOBIELUS, J.}

Avoiding the high-tech sweatshop syndrome. Network World, v. 11, n. 17, p. 32, apr. 1994.

MARTIN, P. Y.;

GLISSON, C.

Perceived structure: welfare organizations in three societal cultures. Organizational Studies, New York, v. 10, n. 3, p. 353-380, 1989.

\section{OCDE.}

Manual de Frascati: medición de las actividades científicas y tecnológicas. Paris, France. 1993. p. 250.

\section{PERROW, C.}

A framework for the comparative analysis of organizations. American Sociological Review, Albany, v. 32, n. 2, p.194-208, apr. 1967.
PETERS, T:;

WATERMAN, R. H.

In search of excellence: Lessons from America's best run companies. New York: Knopf, 1982. p. 360.

PUGH, D. S.;

HICKSON, D. J.

Organizational structure in its context. The Aston Programme I. Westmead: Saxon House, 1976. p. 231.

REICH, R.

The work of nations. London: Simon \& Schuster, 1992. p. 339.

SILVA, J. R. G. et al.

Operadores de call center: inconsistências e desafios da gestão de pessoas. ENCONTRO NACIONAL DA ANPAD, 26., 2002, Salvador. Anais... Salvador: ANPAD, 2002. 1 CD-ROM.

TAYLOR, P.;

BAIN, $P$.

'An assembly line in the head': Work and employee relations in the call centre. Industrial Relations Journal, Oxford, v. 30, n.2, p. 101-117, june 1999.

TOFFLER,A.

A empresa flexível. 7. ed. Rio de Janeiro: Editora Record, 1985. p. 244.

VASCONCELOS, I. F. G. et al.

O lado humano da tecnologia: um estudo exploratório sobre os paradoxos organizacionais dos sistemas de informação. ENCONTRO NACIONAL DA ANPAD, 25., 2001, Campinas. Anais... Campinas: ANPAD, 2001. 1 CD-ROM. 
WOODWARD, J.

Industrial organization: theory and practice. Oxford: Oxford University Press, 1965. p. 281.

\section{ZUBOFF, S.}

In the age of the smart machine. Havard, MA: Harvard Business, 1988. p. 468. 